\title{
A wearable "electronic patch" for wireless continuous monitoring of chronically diseased patients
}

Haahr, Rasmus Grønbek; Duun, Sune; Thomsen, Erik Vilain; Hoppe, Karsten; Branebjerg, Jens

Published in:

5th International Summer School and Symposium on Medical Devices and Biosensors, 2008. ISSS-MDBS 2008.

Link to article, DOI:

10.1109/ISSMDBS.2008.4575018

Publication date:

2008

Document Version

Publisher's PDF, also known as Version of record

Link back to DTU Orbit

Citation $(A P A)$ :

Haahr, R. G., Duun, S., Thomsen, E. V., Hoppe, K., \& Branebjerg, J. (2008). A wearable "electronic patch" for wireless continuous monitoring of chronically diseased patients. In 5th International Summer School and Symposium on Medical Devices and Biosensors, 2008. ISSS-MDBS 2008. IEEE. https://doi.org/10.1109/ISSMDBS.2008.4575018

\section{General rights}

Copyright and moral rights for the publications made accessible in the public portal are retained by the authors and/or other copyright owners and it is a condition of accessing publications that users recognise and abide by the legal requirements associated with these rights.

- Users may download and print one copy of any publication from the public portal for the purpose of private study or research.

- You may not further distribute the material or use it for any profit-making activity or commercial gain

- You may freely distribute the URL identifying the publication in the public portal 


\title{
A Wearable "Electronic Patch" for Wireless Continuous Monitoring of Chronically Diseased Patients
}

\author{
Rasmus G. Haahr, Sune Duun, Erik V. Thomsen, Karsten Hoppe, and Jens Branebjerg
}

\begin{abstract}
We present a wearable health system (WHS) for non-invasive and wireless monitoring of physiological signals. The system is made as an electronic patch where sensors, low power electronics, and radio communication are integrated in an adhesive material of hydrocolloid polymer making it a sticking patch. The patch is made with a reusable part and a disposable part which contains the adhesive material and the battery. This part is changed once every week. The patch has a size of $88 \mathrm{~mm}$ by $60 \mathrm{~mm}$ and a thickness of $5 \mathrm{~mm}$. It is made for attachment on truncus or the greater muscle groups. The patch is demonstrated in two applications: Monitoring of electromyography (EMG) and arterial oxygen saturation by pulse oximetry $\left(\mathrm{SpO}_{2}\right)$. The pulse oximetry sensor is made of a concentric backside Silicon photodiode with a hole in the middle for the two light sources. This makes it suitable for reflectance pulse oximetry. For the EMG application three standard dry silver electrodes are used separated by $10 \mathrm{~mm}$.
\end{abstract}

\section{INTRODUCTION}

During the last decade there have been an increasing interest in new technology and innovative systems for the health care system. Significant factors such as limitations in the health care system's resources, the aging population, and chronic conditions are motivating research.

In this context non-invasive wearable health systems (WHS) for monitoring elderly and chronically diseased people outside hospitals have been developed. Various approaches have taken place for continuously monitoring of vital signs. H. Asada et. al presented in 1998 a system made as a small finger ring sensor [1]. A European project "Sensation" have followed the same the approach [2]. J. Kang et. al have developed an instrument worn on the wrist [3]. Devices attached to truncus have not been made, but sensors have been integrated in textiles or clothes [4], [5]. Following these ideas an European project "Wealthy" have developed an integrated system [6], [7].

The vision for our research is to create a technology which allows for continuously monitoring of elderly and chronically diseased on a 24/7 basis. Furthermore, the sensor system must be able to function for a week without having to be changed. The technology should be convenient, versatile, easy to use, well integrated in the modern health care system and should provide safety and service for the patients.

Manuscript received February 25, 2008. This work was party supported by the Danish Ministry of Science, Technology and Innovation.

Rasmus G. Haahr, Sune Duun, and Erik V. Thomsen are with the Technical University of Denmark, Department of Micro and Nanotechnology, Oersted Plads building 345 east, 2800 Kgs. Lyngby, Denmark (Phone: + 45 45255700; Fax: +45 45887762; e-mail: rasmus.haahr@mic.dtu.dk, sdu@mic.dtu.dk, evt@mic.dtu.dk).

Karsten Hoppe and Jens Branebjerg are with Delta A/S, Venlighedsvej 4, 2970 Hørsholm, Denmark (e-mail: kh@delta.dk, jab@delta.dk).

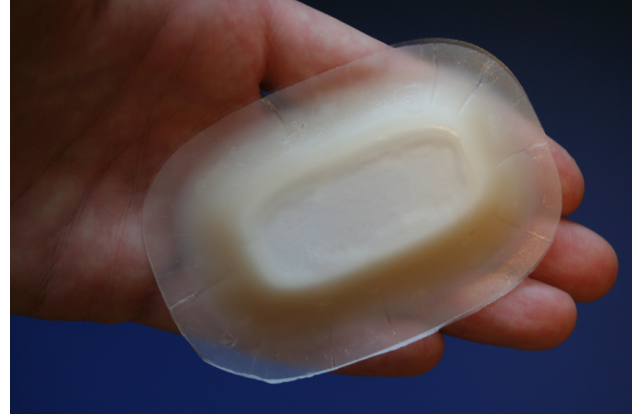

Fig. 1. The "Electronic Patch". The patch has as a size of $88 \mathrm{~mm}$ by 60 $\mathrm{mm}$ and is $5 \mathrm{~mm}$ thick.

In this paper we report the recent development of a patient monitoring system the "Electronic Patch" shown in Fig. 1 which we previously have proposed [8], [9]. The patch can monitor various physiological signals, analyze these and when needed either transmit an alarm or continuously stream data over a wireless network.

In this paper we describe the Electronic Patch in the application of monitoring electromyography (EMG), and pulse oximetry for monitoring the oxygen saturation $\left(\mathrm{SpO}_{2}\right)$ and heart rate. For the latter case we have previously reported a design of a novel ring shaped photodiode [10] which have a concentric detection of light transmitted from two light emitting diodes (LEDs) placed in a hole in the center of the chip.

\section{Possibilities AND Limitations FOR WEARABLE AND NON-INVASIVE MONITORING}

Today clinical care and monitoring of elderly and chronically diseased is typically done by frequent health examinations at the hospital or local physician. A new paradigm for patient monitoring where wearable and wireless systems are introduced to complete routine health examinations induce possibilities for monitoring health conveniently anywhere at all times. In non critical applications it can log physiological information and thereby improve treatment. In critical applications it can automatically transmit an alarm and data if a medical incident is detected.

\section{A. Possibilities}

WHS are preferably non-invasive to limit infection risks. A variety of physiological data can be measured by noninvasive techniques e.g. electrocardiogram (ECG), electromyogram (EMG) electroencephalogram (EEG), blood 
pressure, heart rate, photoplethysmogram (PPG), phonocardiogram (PCG), oxygen saturation $\left(\mathrm{SpO}_{2}\right)$, saturation of carbon monoxide (SpCO), skin temperature, respiration frequency, respiration volume, sweat production, tissue perfusion, and motion. There are several measuring principles that can be used for non-invasive monitoring of such physiological signals.

1) Electrical: Electric potentials are used for ECG, EMG and EEG and used for monitoring the hearth and nervous systems. Impedance measurements can be used for monitoring the respiration frequency and resistance for monitoring the skin temperature.

2) Optical: Optical measurements with non-coherent light is typically based on absorption for measuring PPGs, whereas coherent light can be used for laser Doppler bloodflow monitoring. PPGs have had an increased interest in the recent years due to potential of extracting more physiological parameters such as blood pressure [11], [12].

3) Mechanical: Mechanical sensors can be used for measuring respiration volume, motion, blood pressure, phonocardiogram (PCG), and ultrasound based measurements.

4) Chemical: Chemical measurements are typically invasive and have therefore limited possibilities in non-invasive systems. However, there are electrochemical sensors that measure $\mathrm{CO}_{2}$ and $\mathrm{O}_{2}$ transcutaneously. Analysis on sweat is also possible, but this is not clinical practice today.

\section{B. Limitations}

In general WHS are limited by two main factors: Comfort and power consumption. An additional third factor is motion artefacts which is a well-know problem for some types of measurements e.g. ECG and PPG.

For WHS to work on a $24 / 7$ basis attached to people living an everyday life the attachment must neither be physical nor visual annoying for the person so that the person is comfortable wearing it. WHS should therefore be biocompatible and must not cause pressure to the tissue which will prevent blood circulation.

The size of the systems is obviously also a limiting factor and in general WHS should be as small as possible and follow natural body shapes to provide most comfort. In the case WHS are attached directly to the body they must therefore be as flat as possible and not thicker than a few millimeters. Alternatively, WHS could be integrated in things that people would wear anyway e.g. clothes. Typical locations for attaching WHS are the finger or wrist e.g. [1], [3]; however, these locations are not convenient in daily activities. Locations on truncus hidden by clothes seems more attractive. These locations have previously been utilized in systems where sensors are incorporated in clothes [4], but this approach does not offer a firm attachment of the sensors directly to the body.

The power consumption for WHS is a critical issue and is limited either by the battery or by the amount of energy that can be harvested from the body e.g. thermoelectrically. Since the size of WHS should be as small as possible the systems cannot have large batteries and due to convenience

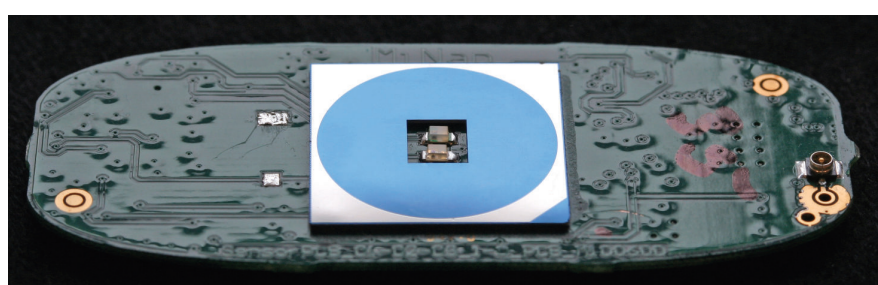

Fig. 2. Ring shaped photodiode with LEDs in the center mounted on bottom side of PCB.

they should last as long as possible. Power is consumed by the sensors, frontend electronics, digital processing of data, and radio communication.

Sensors for some physiological signals require very little power e.g. potential measurements as EMG. But other physiological signals require more energy consuming sensors e.g. pulse oximetry due the driving current for the LEDs. For some applications it is therefore necessary to focus both on the energy consumed by the sensors as well as the successive frontend electronics and digital signal processing.

Power management can be done by several approaches. In general radio communication should be limited since this is highly power consuming. It is therefore advantageous to have signal analysis done in the WHS and then only transmit data or just an alarm when abnormalities arise.

The nature of the physiological signals should also be considered. Some change in the scale of hours e.g. temperature some change in the scale of minutes e.g. oxygen saturation and some change in the scale of seconds e.g. the status of the heart as monitored by ECG. The requirement to the measuring frequency is therefore different between these types of signals. By measuring quasi continuously with a period corresponding to the natural period of the change in the signals power can be conserved without losing critical information.

\section{Overview of the Electronic Patch System}

Based on the following considerations we have developed a WHS as an electronic patch. The Electronic Patch is a genuine platform which is compatible with many types of sensors. In this paper we describe two applications: monitoring of EMG and $\mathrm{SpO}_{2}$ by pulse oximetry. The EMG sensor is intended for detection of convulsions during sleep and the pulse oximetry sensor is intended for people suffering from heart disorders, chronical lung diseases (COLD), sleep apnea, and professionals during work such as fire fighters.

The Electronic Patch consists of a printed circuited board (PCB) where sensors are mounted on the bottom, and the top contains all the electronics and radio communication. The PCB is encapsulated in a hard plastic box and attached to the body by an adhesive material of hydrocolloid polymer.

\section{A. Sensors}

The EMG sensor have a standard design made by three silver electrodes distributed evenly on the PCB with a separation of $10 \mathrm{~mm}$. The pulse oximetry sensor comprises a concentric photodiode with two LEDs in the middle a red 


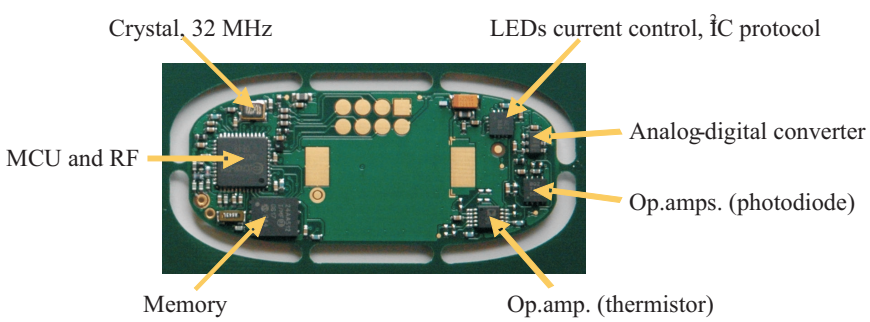

Fig. 3. Top side of the PCB showing the types of electronic components which is utilized in the pulse oximetry version of the Electronic Patch.

$(660 \mathrm{~nm})$ and infrared $(940 \mathrm{~nm})$ [10]. The sensor is shown in Fig. 2.

\section{B. Electronics}

The top side of the PCB contains the electronics as shown in Fig. 3. It contains analog frontend electronics, a low power microprocessor with a built-in radio, and memory. The microprocessor uses from $190 \mu \mathrm{A}$ at $32 \mathrm{kHz}$ with the radio off to $27 \mathrm{~mA}$ at $32 \mathrm{MHz}$ with the radio on. The power usage of the microprocessor will be application dependent. In the pulse oximetry sensor we also have an $\mathrm{I}^{2} \mathrm{C}$ current controller to control the LEDs. The patch is powered by a coin size 3 V Lithium-ion battery with $170 \mathrm{mAh}$.

\section{Wireless communication and network}

The wireless networking in the Electronic Patch is based on a $2.4 \mathrm{GHz}$ radio and a proprietary protocol which allows the patch to work in a wireless personal area network, but not as an independent system in direct contact with service providers or hospitals. However, this contact can be made by external access points connected to the internet e.g. smart phones. Access points could also be installed in the person's home or other daily environments. The advantage using this solution is that power consuming long distance communication is placed outside the patch. This configuration also supports the service of many patches. For instance in the case of assisted living homes where many elderly could be monitored by individual patches each connected to the same network of access points covering the entire estate. A proprietary protocol has been employed instead of the ZigBee and Bluetooth protocols due to lower power consumption. The drawback is a limited range of a few meters. This would be increased by using the Bluetooth protocol.

\section{Mechanical assembly}

The mechanical assembly is shown in Fig. 4 and the final patch with the pulse oximetry sensor is shown in Fig. 5. Sensors and electronics are encapsulated in a bio-compatible plastic housing which protect the electronics from sweat and moisture. The pulse oximetry sensor is further protected by a transparent membrane and the EMG sensor has an epoxy seal. With this solution the system can even be warn during a shower.

The patch comes in two parts: 1) A reusable sensor part consisting of a bottom- (f) and middle plastic housing (d),

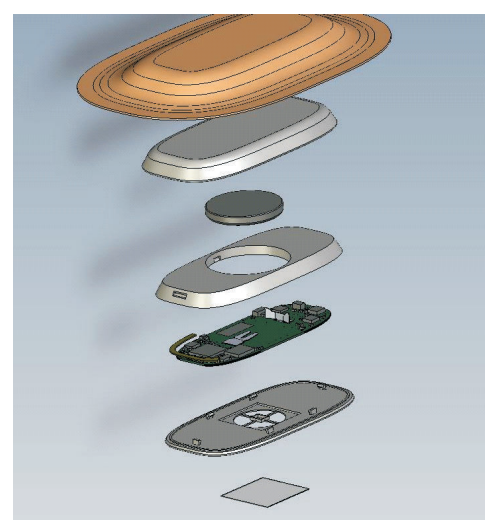
(a) Adhesive patch
(b) Plastic housing - top
(c) Battery
(d) Plastic housing- middle
(e) Printed circuit board PCB
(f) Plastic housing - bottom
(g) Bio-compatible "window"

Fig. 4. CAD drawing of the parts in the electronic patch and how they are assembled.

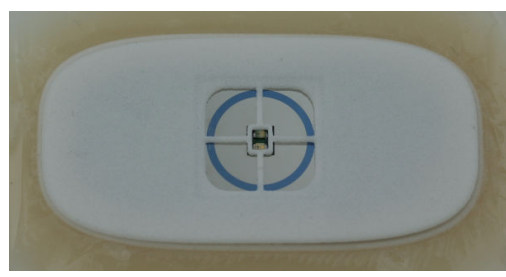

Fig. 5. The assembled patch with a pulse oximetry sensor made as a concentric photodiode around two LEDs placed in the center. The little square frame around the LEDs is to prevent light going directly from the LEDs into the photodiode.

sensors and electronics (e). 2) A disposable part consisting of the adhesive patch (a), top housing (b), and battery (c). The adhesive patch has to be changed once every week due to dead skin cells. This is therefore the period which the battery has been designed to last. The adhesive patch is designed for attaching the plastic housing onto the skin and the hydrocolloid polymer allows for diffusion of moisture away from the skin.

\section{EMG APPLICATION}

Electromyography is a method of detecting muscle activity. The methods relies on the change of membrane potential of the muscle cells with muscle activity. The resting muscle cell have a potential across the cell membrane of approximately $-90 \mathrm{mV}$. During muscle activity the membrane potential change to approximately $15 \mathrm{mV}$. This can occur both in spikes when the muscle is stimulated or constantly when the muscle contraction is tetanic. EMG can be measured both non-invasively on the skin surface above the muscle or invasively by needles.

We have used a standard configuration for surface EMG where the potential is measured between two electrodes relative to a third electrode placed in between. The measured signal is amplified, and to save power an analog circuit for detection of spikes have been employed. The microprocessor is then only turned on whenever spikes are detected and the muscle is active. This is demonstrated in Fig. 6 where the yellow (top) curve is the recorded EMG signal and 


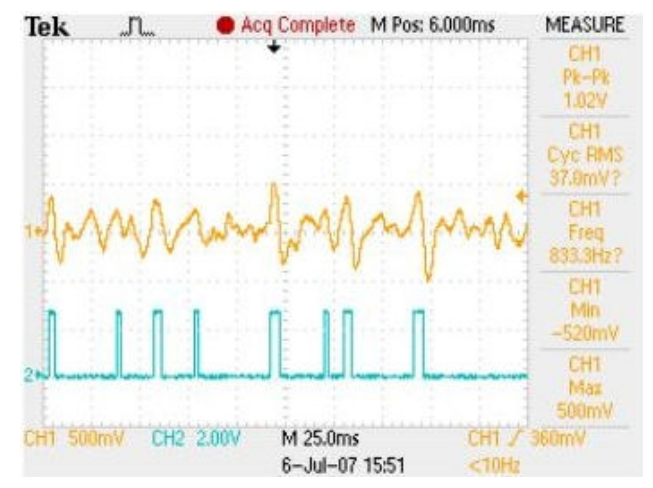

Fig. 6. The yellow (top) graph shows a recorded EMG signal by the Electronic Patch. The blue (bottom) curve indicates the turn on and off of the microprocessor which is controlled by an analog frontend spike detecting circuit.

the blue (lower) curve indicates the turn on and off of the microprocessor. The microprocessor then analyze the EMG signal and evaluate if convulsions are taking place.

\section{Pulse Oximetry Application}

A pulse oximetry sensor detects pulse and arterial oxygen saturation. It is an optical technique invented by T. Aoyagi in 1972 [13] and is based on absorption changes of light with the blood flow. Pulse oximetry relies on the difference in the absorption spectra between oxygenated haemoglobin $\left(\mathrm{HbO}_{2}\right)$ and deoxygenated haemoglobin $(\mathrm{Hb})$. In [9] it is shown that the ratio between absorption coefficients of $\mathrm{HbO}_{2}$ and $\mathrm{Hb}$ makes wavelengths of $660 \mathrm{~nm}$ and $940 \mathrm{~nm}$ suitable.

For the pulse oximetry application we have chosen to custom design pn Silicon photodiodes. This allows for optimization of the photodiodes for the pulse oximetry application. To minimize the necessary driving current of the LEDs we have fabricated large area photodiodes which are concentric around the LEDs and hence optimized for collection of backscattered light from the tissue [10]. This approached was pioneered by Y. Mendelson [14]; however, with the use of several discrete photodiodes. The photodiodes have a chip size of $14 \mathrm{~mm}$ by $14 \mathrm{~mm}$ and with various active areas ranging from $22 \mathrm{~mm}^{2}$ to $121 \mathrm{~mm}^{2}$. This area is up to 20 times larger than what is used in a Nellcor wired reflectance sensor. The largest photodiode is shown in Fig. 2. We have described the fabrication process elsewhere [10].

Increasing the photodiode area also increases the capacitance and this will lower the speed of the photodiode, hence there is a tradeoff between photodiode area and speed. In our system we use a sampling rate, $f_{\mathrm{s}}$, of $1 \mathrm{kHz}$. The capacitances of the largest photodiodes are $24 \mathrm{nF} \pm 2 \mathrm{nF}$. Given a photodiode transimpedance amplifier circuit with a $10^{4}$ amplification the bandwidth, $B W$, will approximately be given by:

$$
B W \simeq\left(C_{\mathrm{PD}} \cdot R_{\mathrm{Amp}}\right)^{-1}=(24 \mathrm{nF} \cdot 10 \mathrm{k} \Omega)^{-1}=4 \mathrm{kHz}
$$

We have fabricated several $1 \mathrm{~mm}$ wide rings with radii from $3.5 \mathrm{~mm}$ to $6.5 \mathrm{~mm}$. This is done to gain knowledge about at what radii on a specific body location the signal

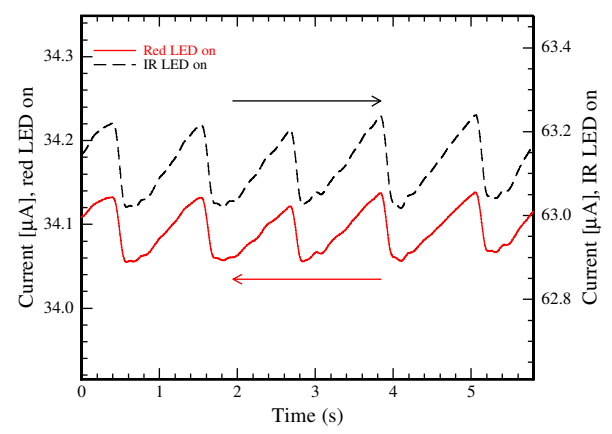

Fig. 7. Photoplethysmograms measured with Electronic Patch pulse oximetry sensor

has the best signal to noise ratio. One such ring sensor is seen in Fig. 5.

To ease the assembly we have chosen to make backside photodiodes which have the junction and both contacts on the side facing the PCB. Therefore, no wirebonding is necessary.

To shield from ambient light and to optimize transmission at the two wavelengths of interest i.e. $660 \mathrm{~nm}$ and $940 \mathrm{~nm}$ a two layer antireflection filter consisting of $550 \mathrm{~nm}$ PECVD Silicon Nitride on $50 \mathrm{~nm}$ thermal dry Silicon Oxide has been employed. This filter reach optical transmission $>98 \%$ at $660 \mathrm{~nm}$ and $940 \mathrm{~nm}$ and suppressing other wavelengths to approximately $50 \%$ in the range $600 \mathrm{~nm}-1100 \mathrm{~nm}$. For wavelengths below $600 \mathrm{~nm}$ the tissue absorbtion is very strong and hence ambient light at these wavelengths does not course problems. The photodiodes are also patterned with Aluminum on the side of the light entrance to give a welldefined area of light gathering.

Photopletsysmograms like the one shown in Fig. 7 have been recorded with the fabricated sensor. From the PPGs the pulse and the oxygen saturation can be calculated.

To further optimize the power consumption of the pulse oximetry sensor the duty cycle of the LEDs, $D_{\text {LED }}$, can be considered. The minimal duty cycle that is possible, when at least $95 \%$ of the LED power must be maintained, is given by the sampling frequency and the bandwidth of the photodiode amplifier circuit [15]. In our case we get

$$
D_{\mathrm{LED}} \simeq 2 \cdot f_{\mathrm{s}} / B W=2 \cdot 1 \mathrm{kHz} / 4 \mathrm{kHz}=50 \%
$$

When lit the LEDs typically use $20 \mathrm{~mA}$ at $1.5 \mathrm{~V}$. The $\mathrm{I}^{2} \mathrm{C}$ current controller needs $10 \mathrm{~mA}$ at $3 \mathrm{~V}$ to deliver $20 \mathrm{~mA}$ at $1.5 \mathrm{~V}$. Having a duty cycle of $50 \%$ on the LEDs the $\mathrm{I}^{2} \mathrm{C}$ current controller on average will use $5 \mathrm{~mA}$ at $3 \mathrm{~V}$. If we measure continuously the LEDs alone would use the battery in 34 hours. Therefore, we would like to reduce the LED power consumption by at least a factor of 10 . Because then we can measure continuously for a week and only use 85 $\mathrm{mAh}$ or half the battery power available on the LEDs. One way to do this will be to improve the speed of the photodiode amplifier circuit by lowering the photodiode capacitance.

\section{DISCUSSION}

The Electronic Patch have at this time been tested in the laboratory and on persons wearing it for periods of one week. 
Tests in a clinical setting have not been done at this time. Thus there are still questions to be examined regarding longterm use and actual benefits compared to traditional health monitoring and other wearable health monitoring systems. Likewise clinical experiments is also required to calibrate the pulse oximeter. However, this is a standard procedure.

In contrast to other systems attached around the finger, wrist or integrated in clothes the Electronic Patch is mounted directly to the truncus or greater muscles groups offering a very firm attachment of the sensors and no wiring between several units. The Electronic Patch is therefore a versatile system and very simple to use.

The reliability of WHS are both dependent on the attachment of the sensors and the algorithms used to analyze the signals. Development of algorithms which provide enough and reliable artificial intelligence are therefore important.

The most suitable locations to measure depend on the application. For the EMG application the location must be the on muscle which is being examined. Typically, this will be the larger muscle groups e.g. biceps. In case of the pulse oximetry application further research have to be done in this direction. The Electronic Patch is not compatible with the typical locations for pulse oximetry i.e. the finger or forehead since this will be visually annoying for the patient. We are targeting a location on truncus either ventral or dorsal and we are currently investigating this. Pulse oximetry on the sternum have previously been demonstrated by [16], but it needs more investigation before it can be used clinically.

The most suitable wireless network to use in WHS dependent on the application. If WHS are intended for continuously monitoring the WHS should be able to get online often and the power consumed by the radio is therefore important. However, in the applications where WHS only should transmit an alarm when a critical condition arises it is more important that the WHS can get online. Since it only need to transmit once it can use more power and in this case the global system for mobile communications (GSM) network could be employed.

As we mention in this paper the large capacitances of the large area photodiodes impose a problem regarding the speed. This could be improved by pin photodiodes. Our fabrication process for the photodiodes are compatible with a step where an epitaxial layer of Silicon is grown making the photodiodes of the pin type.

\section{CONCLUSION}

We have developed an Electronic Patch as a wearable health monitoring system. The Electronic Patch is a genuine platform which can be used with many types of sensors. The Electronic Patch is demonstrated in two very different applications EMG and pulse oximetry monitoring. These applications rely on two different measuring principles i.e. electrical and optical.

For the pulse oximetry application a novel concentric photodiode is used which lower the requirement to the LED driving current. For the EMG application standard silver electrodes are used. The electronics in the Electronic Patch are low power surface mountable components. The patch is powered by a $3 \mathrm{~V}$ Lithium-ion battery which last a week and is changed when the adhesive material needs to be changed anyway.

We will fabricate pin photodiodes in order to reduce the capacitance of the photodiodes which will allow for a lower duty cycle of the LEDs. This will reduce the power consumption of the pulse oximetry sensor.

The Electronic Patch is made compatible with wireless personal area network; however, the patch also relies on external devices for online communication with service providers or hospitals. The EMG and pulse oximetry application sensors were shown to work, but clinical test and trials of the Electronic Patch still remain to be investigated.

\section{REFERENCES}

[1] H. Asada, "The Ring Sensor: a New Ambulatory Wearable Sensor for Twenty-Four Hour Patient Monitoring", Proc. 20th Annual IEEE EMBS conf., vol. 4, pp 1906-1909, 1998

[2] J. Sola el. al, "SpO2 Sensor Embedded in a Finger Ring: desing and implementation" Proc. 28th Annual IEEE EBMS conf. , pp 4295-4298, 2006

[3] J. M. Kang, T. Yoo, H. C. Kim, "A wrist-worn integrated health monitoring instrument with a tele-reporting device for telemedicine and telecare", IEEE Transactions on Instrumentation and Measurement, vol. 55, nr. 5, pp 1655-1661, 2006

[4] A. D. Santa, A. Mazzoldi, D. De Rossi, "Dressware: warable hardware" Mater Sci Eng, C7:31.37, 1999

[5] G. Loriga, N. Taccini, D. De Rossi, R. Paradiso, "Textile Sensing Interfaces for Cardiopulmonary Signs Monitoring" Proc. 27th Annual IEEE EBMS conf. , pp 7349-7352, 2005

[6] R. Paradiso, G. Loriga, N. Taccini, "Wearable System for Vital Signs Monitoring" Wearable eHealth Systems for Personalised Health Management, pp 253-259, 2004

[7] R. Paradiso, G. Loriga, N. Taccini, "A Wearable Health Care System Based on Knitted Integrated Sensors" IEEE Transactions on Information Technology in Biomedicine, vol. 9, no. 3, pp 337-344, 2005

[8] E. V. Thomsen, A. Hyldgård, K. Birkelund, R. G. Haahr, S. Duun, R. Elsubaihi, J. Branebjerg, "Distributed sensors: Applciations, Fabrication and Challenges" Proc. 20th Eurosensors Conf., 2006.

[9] R. G. Haahr, S. Duun, K. Birkelund, P. Raahauge, P. Petersen, H. Dam, L. Nørgaard, E. V. Thomsen, "A Novel Photodiode for Reflectance Pulse Oximetry in low-power applications" Proc. 29th Annual IEEE EMBS conf., pp. 2350 - 2353, 2007

[10] S. Duun, R. G. Haahr, K. Birkelund, P. Raahauge, P. Petersen, H. Dam, L. Nørgaard, E. V. Thomsen, "A Novel Ring Shaped Photodiode for refelctance Pulse Oximetry in Wireless Applications" Proc. 6th Annual IEEE Sensors Conf., pp. 596 - 599, 2007

[11] X. F. Teng, Y. T. Zhang, "Continous and Noninvasive Estimation of Arterial Pressure Using a Photoplethysmographic Approach" Proc. 25th Annual IEEE EBMS conf. , pp 3153-3156, 2003

[12] P. A. Shaltis, A. Reisner, H. H. Asada, "Wearable, Cuff-less PPGBased Blood Pressure Monitor with Novel Height Sensor" Proc. 28th Annual IEEE EBMS conf. , pp 908-911, 2006

[13] T. Aoyagi, M. Kishi, K. Yamaguchi, S. Watanabe. "Improvement of the earpiece oximeter", Abstracts of the 13th Annual Meeting of the Japanese Society of Medical Electronics and Biological Engineering, pp 90-91, 1974 (Jap)

[14] Y. Mendelson, "Design and evaluation of a new reflectance pulse oximeter sensor", Medical Instrumentation, vol. 22, nr. 4, pp 167173,1988

[15] E. A. Peláez and E. R. Villegas, "LED power reduction trade-offs for ambulatory pulse oximetry"Proc. 29th Annual IEEE EMBS conf., pp. $2296-2299,2007$.

[16] J. Sola, O. Chetelat, J. Krauss, "One the reliability of pulse oximery at the sternum" Proc. 29th Annual IEEE EBMS conf. , pp 1537, 2007 